\title{
A Right-sided "J" -shaped Incision Provides Full Exposure of a Penetrating Wound from the Left Anterior Neck to the Right Thoracic Cavity: A Case Report
}

\author{
Jung Joo Hwang ${ }^{1}$, Jin Ho Choi ${ }^{1}$, Min Seok Choi ${ }^{1}$, Dongsub Noh', Chan Yong Park', \\ Hyun Min $\mathrm{Cho}^{2}$
}

'Department of Trauma \& Cardiothoracic Surgery, Eulji University Hospital, Daejeon, Korea

${ }^{2}$ Department of Trauma Surgery, Pusan National University Hospital, Busan, Korea

We present a case report of a penetrating neck injury from the anterior neck to the right thoracic cavity. When we encounter such a case in the operating room, it is difficult to decide on an approach that exposes the entirety of the wound. In this case, only a partial sternotomy with a right anterior thoracotomy (extended "J" -shaped incision) provides full exposure of the injured area. Such an incision may be very effective in selective cases.

Key Words: Neck injury; Sternotomy; Thoracotomy

(Trauma Image Proced 2017(2):59-61)

\section{CASE}

A 77-year-old man presented to the trauma bay with a self-inflicted neck stab wound (Fig. 1). Initial measurements of his vital signs revealed a blood pressure of $187 / 110 \mathrm{mmHg}$, pulse rate of 59 beats/min, respiration rate of 22 breaths $/ \mathrm{min}$, body temperature of $36.0^{\circ} \mathrm{C}$, and oxygen saturation of $97 \%$. He was drunk, and we could not check his mental state. The patient had a previously installed pacemaker because of a left bundle branch block. Initial chest X-rays showed that a knife traversed from the anterior region of the neck to the right thoracic cavity and induced a right pneumothorax (Fig. 2). A chest computed tomography (CT) scan showed a left lower neck laceration, thyroid gland injury, a foreign body in the mediastinum and the right hilum, and the tip was a posterior arc of the 6th rib (Fig. 3). We could not rule out injuries to the thyroid gland, trachea, and brachiocephalic and subclavian arteries and thus elected to perform an emergency operation. We performed a partial sternotomy and then extended to the right anterior thoracotomy. This incision looked like an extended "J" shape. With this incision, we could see the full injury area and were thus able to remove the knife and repair the lung, pleura, and thyroid and ligate small mediastinal vessels (Fig. 4). The patient was discharged on postoperative day 7 without any complication (Fig. 5).

Received: November 6, 2017 Revised: November 10, 2017 Accepted: November 10, 2017

Correspondence to: Jung Joo Hwang, Department of Trauma \& Cardiothoracic Surgery, Eulji University Hospital, 95, Dunsanseo-ro, Seo-gu, Daejeon, Korea

E-mail: drthora1@naver.com

Copyright (c) 2017 Korean Association for Research, Procedures and Education on Trauma. All rights reserved.

@This is an open-access article distributed under the terms of the Creative Commons Attribution Non-Commercial License (http://creativecommons.org/ licenses/by-nc/4.0) which permits unrestricted noncommercial use, distribution, and reproduction in any medium, provided the original work is properly cited 


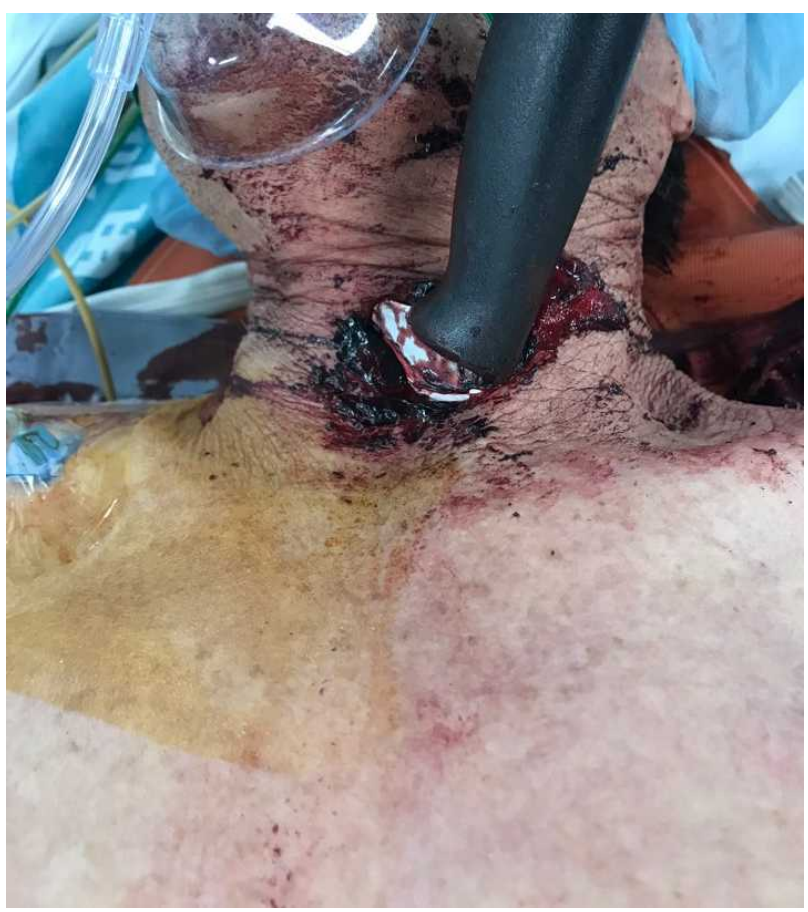

Fig. 1. The knife handle in the left anterior neck area

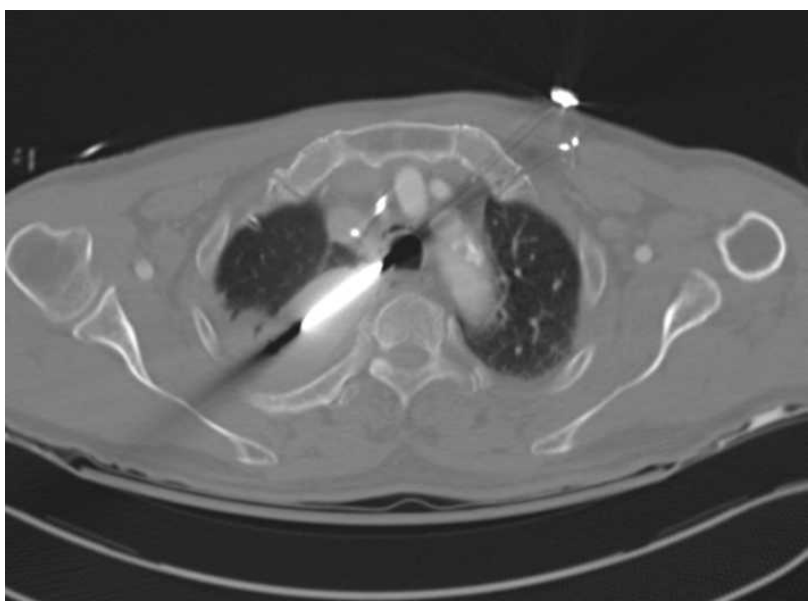

Fig. 3. Chest CT shows a foreign body in the left thoracic cavity and a right hemothorax and a laceration of the right-upper region of the lung.

\section{DISCUSSION}

Penetrating neck injuries occur in approximately 5$10 \%$ of all trauma patients who present to the emergency room. About $30 \%$ of these neck injuries are accompanied by trauma outside the neck zones as

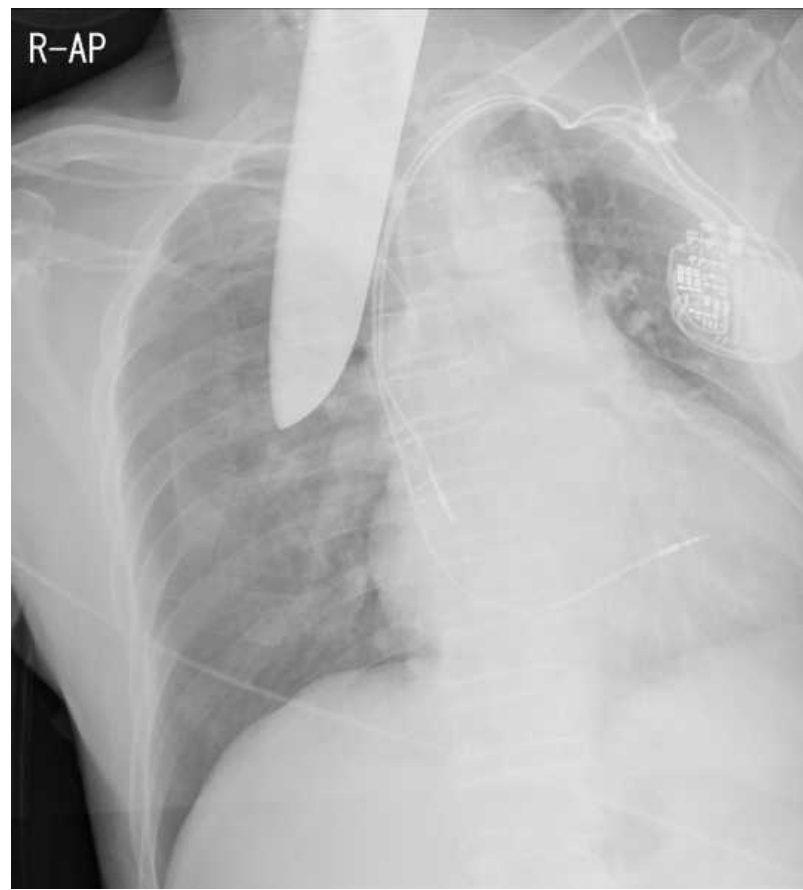

Fig. 2. Simple chest $X$-ray shows a right pneumothorax and a knife from the left anterior region of the neck to the right thoracic cavity.

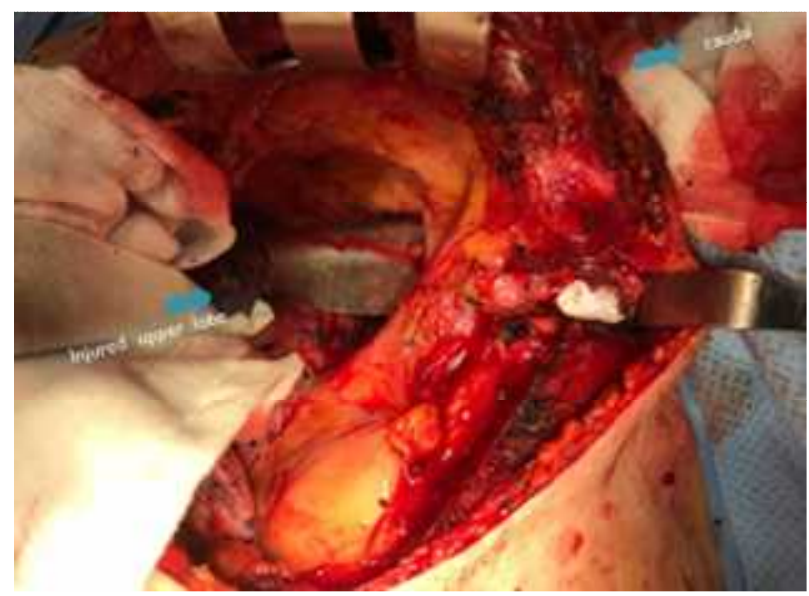

Fig. 4. Extended "J- shaped incision exposes major injuries

observed in the present case $(1,2,3)$. In the presence of right-sided lung and vascular injuries, the common surgical approach typically involves median sternotomy. With this incision, sometimes it is difficult to inspect the full injuries of the vessels and thoracic organs; therefore, a sternotomy with an extension of the anterior 


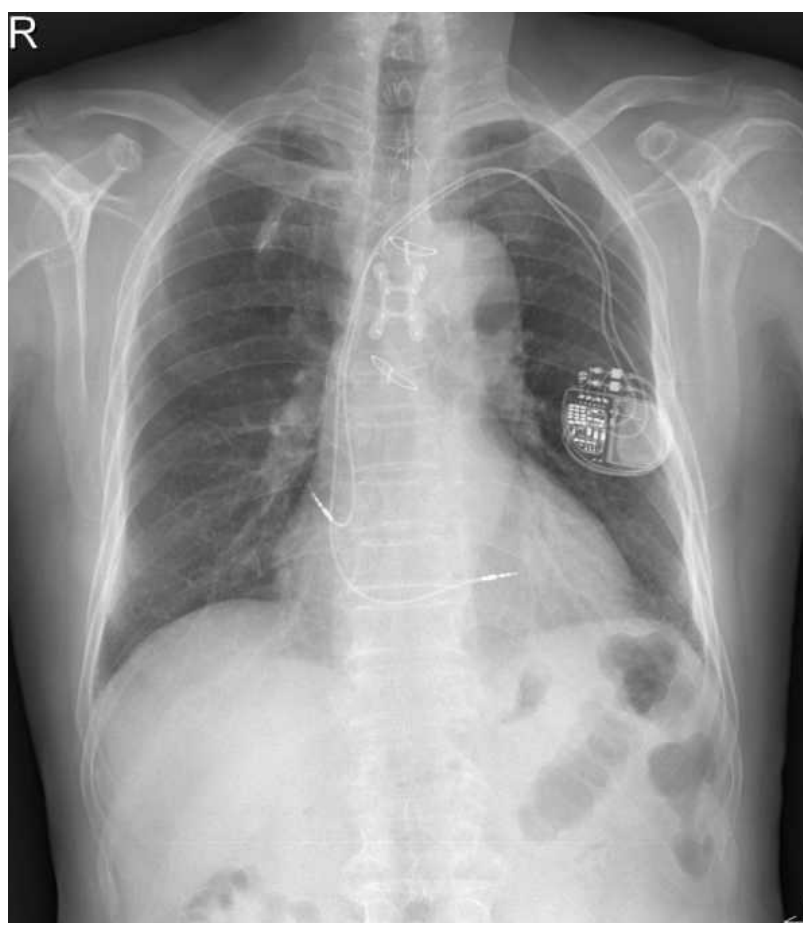

Fig. 5. Chest $\mathrm{X}$-ray 1 month after operation

thoracotomy may be more useful in such situations. However, it is also known that a combination of an anterolateral thoracotomy, partial sternotomy, and left infra- or supraclavicular incision described as "trap-door" thoracotomy is rarely performed due to the time requirements and because it results in multiple fractures (4). In the present case, the injury encompassed regions from left cervical zone 1 to the right thoracic cavity and the vital signs were stable. First, we planned to make a "trap-door" incision because subclavian vessel injuries could not be ruled out; however, after division of the manubrium and 3rd intercostal space, there were no subclavian vessel injuries. Thus, we were able to remove the knife, repair the lacerated lung, ligate the transected branches of the subclavian vessels, and repair the thyroid through this incision. During trauma surgery, it is challenging to expose arch vessels, their branches, and mediastinal structures; therefore, the selection of the incision depends on mediastinal structures that need to be explored during surgery $(5,6)$. The "trap-door" incision, or a modified version of this method, is an option in such cases.

\section{Conflict Of Interest}

This work was supported by clinical research grant from Pusan National University Hospital in 2016.

\section{REFERENCES}

1. McConnell DB, Trunkey DD. Management of penetrating trauma to the neck. Advances in surgery. 1994;27:97-127.

2. Cho, Hyun Min. "How Do I Manage Penetrating Neck Trauma?" Trauma Image Proced 2, no. 1 (5 2017): 1-6.

3. Park, Chan Yong, Hyun Min Cho, and Seok Ran Yeom. "Tracheal Injury Caused by Self-Stabbing over the Low Anterior Neck." Trauma Image Proced 2, no. 1 (5 2017): 35-39

4. Boffard K: Manual of Definitive Surgical Trauma Care, Second Edition. International Association for Trauma Surgery and Intensive Care. Hodder Arnold. Part 4. Specific Organ Injury. 2007:page90.

5. Fabregues Olea, A., L. Zarain Obrador, D. Perez-Diaz, and F. Turegano Fuentes. "Trap-Door Incision for Penetrating Thoracic Trauma: An Obsolete Approach?" Case Rep Surg. $2014: 3$.

6. Kessel, B., I. Ashkenazi, I. Portnoy, D. Hebron, D. Eilam, and R. Alfici. "Right-Sided "Trapdoor" Incision Provides Necessary Exposure of Complex Cervicothoracic Vascular Injury: A Case Report." Scand J Trauma Resusc Emerg Med. 2009; 17: 46. 